\title{
A novel splice variant of XIAP-associated factor 1 (XAF1) is expressed in peripheral blood containing gastric cancer-derived circulating tumor cells
}

\author{
Keiichi Hatakeyama Yushi Yamakawa Yorikane Fukuda $\cdot$ Keiichi Ohshima \\ Kanako Wakabayashi-Nakao • Naoki Sakura • Yutaka Tanizawa • \\ Yusuke Kinugasa $\cdot$ Ken Yamaguchi $\cdot$ Masanori Terashima $\cdot$ Tohru Mochizuki
}

Received: 12 April 2014/Accepted: 23 August 2014/Published online: 13 September 2014

(C) The International Gastric Cancer Association and The Japanese Gastric Cancer Association 2014

\begin{abstract}
Background XIAP-associated factor 1 (XAF1) is ubiquitously expressed in normal tissues, but its suppression in cancer cells is strongly associated with tumor progression. Although downregulation of XAF1 is observed in tumors, its expression profile in the peripheral blood of cancer patients has not yet been investigated. Here, we identified a novel XAF1 splice variant in cancer cells and then investigated the expression level of this variant in peripheral blood containing gastric cancer-derived circulating tumor cells (CTCs).
\end{abstract}

K. Hatakeyama and Y. Yamakawa equally contributed to this work.

Electronic supplementary material The online version of this article (doi:10.1007/s10120-014-0426-3) contains supplementary material, which is available to authorized users.

K. Hatakeyama $\cdot$ Y. Fukuda $\cdot$ K. Ohshima $\cdot$

K. Wakabayashi-Nakao $\cdot$ N. Sakura $\cdot$ T. Mochizuki

Medical Genetics Division, Shizuoka Cancer Center Research

Institute, Shizuoka, Japan

Y. Yamakawa $\cdot$ Y. Tanizawa $\cdot$ M. Terashima $(\bowtie)$

Division of Gastric Surgery, Shizuoka Cancer Center Hospital,

1077 Shimonagakubo, Nagaizumi-cho, Sunto-gun,

Shizuoka 411-8777, Japan

e-mail: m.terashima@scchr.jp

Y. Yamakawa · Y. Kinugasa

Division of Colon and Rectal Surgery, Shizuoka Cancer Center

Hospital, Shizuoka, Japan

Present Address:

Y. Fukuda

G\&G Science, Fukushima, Japan

K. Yamaguchi

Shizuoka Cancer Center Hospital and Research Institute,

Shizuoka, Japan
Methods To identify splice variants, RT-PCR and DNA sequencing were performed in mRNAs extracted from many cancer cells. We then carried out quantitative RTPCR to investigate expression in peripheral blood from all 96 gastric cancer patients and 22 healthy volunteers.

Results The XAF1 variant harbored a premature termination codon (PTC) and was differentially expressed in highly metastatic cancer cells versus the parental cells, and that nonsense-mediated mRNA decay (NMD) was suppressed in the variant-expressing cells. Furthermore, splice variants of $X A F 1$ were upregulated in peripheral blood containing CTCs. In XAF1 variant-expressing patients, the expression levels of other NMD-targeted genes also increased, suggesting that the NMD pathway was suppressed in CTCs.

Conclusions Our study identified a novel splice variant of $X A F 1$ in cancer cells. This variant was regulated through the NMD pathway and accumulated in NMD-suppressed metastatic cancer cells and peripheral blood containing CTCs. The presence of XAF1 transcripts harboring the PTC in the peripheral blood may be useful as an indicator of NMD inhibition in CTCs.

Keywords Alternative splicing - Circulating tumor cells · Gastric cancer - Nonsense-mediated mRNA decay . Quantitative real-time polymerase chain reaction (qRTPCR)

\section{Introduction}

XIAP-associated factor 1 (XAF1) has been identified as a nuclear protein and a binding partner that directly interacts with endogenous X-linked inhibitor of apoptosis (XIAP) [1]. XAF1 overexpression induces apoptosis and inhibits 
tumor growth in multiple types of cancer including gastric, colorectal, and pancreatic cancers [2-7]. XAFl is ubiquitously expressed in normal cells but expressed at extremely low levels in several types of cancer cells [8]. Lower expression of this gene in tumor tissues is strongly associated with tumor stage $[2,5,9,10]$. Splice variants of $X A F 1$ have been detected in various cancer cell lines [1113]. Fang et al. [14] found a switch from full-length to short XAF1 transcripts in prostate cancer cells, suggesting differential function of the short variant in apoptosis regulation. The production of these transcripts is regulated through aberrant epigenetic modification [12-14]. However, the expression profile of $X A F 1$ splice variants in human cancer remains unclear.

Nonsense-mediated RNA decay (NMD) helps the cell to maintain mRNA quality [15]. Abnormal transcripts generated by alternative splicing often harbor premature termination codons (PTC), leading to degradation of these transcripts via the NMD pathway. NMD is suppressed by cellular stresses in the tumor microenvironment, the inhibition of which promotes stabilization of NMD-targeted mRNA and tumorigenesis [16-19]. Recently, Tani et al. [20] reported that accumulation of noncoding RNA growth-arrest-specific 5 (GAS5) by NMD inhibition through cellular stress (such as serum starvation) leads to the downregulation of apoptosis-related genes. Thus, aberrant RNAs that accumulate through NMD inhibition are considered to be potential tumor markers or biomarkers.

Although a meta-analysis of the published literature revealed that circulating tumor cells (CTCs) are involved in the poor prognosis of gastric cancer patients [21], the associated gene expression profiles remain unclear. Epithelial-mesenchymal transition (EMT)-related genes have been shown to be often expressed in CTCs [22-24], and several genes are abnormally spliced in the EMT [25-27]. RNA-Seq analysis revealed that alternative splicing can induce critical aspects of EMT-associated phenotypic changes, suggesting that the EMT is closely related to RNA splicing [28]. Recently, Valacca et al. [29] reported that aberrantly spliced transcripts accumulated as a result of NMD inhibition in an in vitro model of the EMT. Thus, CTCs in which the EMT is occurring may demonstrate alternative splicing that generates transcripts which would normally be targeted by NMD.

Quantitative real-time polymerase chain reaction (qRTPCR) is one of the most sensitive methods for evaluation of gene expression and is utilized to detect mRNA tumor markers in peripheral blood, such as mRNA encoding cytokeratin 19 (CK19), cytokeratin 20 (CK20), and carcinoembryonic antigen (CEA; synonym, CEACAM5) [3032]. The PAXgene qRT-PCR assay can detect stabilized RNA in the peripheral blood, thus reflecting the expression level of transcripts in CTCs, which showed strong concordance with the results of CTC counting by immunomagnetic separation (CellSearch; Janssen Diagnostics, Raritan, NJ, USA) [33, 34]. However, several markers detected in peripheral blood are frequently expressed in normal epithelial cells, resulting in decreased sensitivity and specificity of qRT-PCR [35]. To maintain the performance of this method using peripheral blood samples, cancer-specific transcripts must be selected [34, 36].

In this study, to evaluate the potential utility of a splice variant harboring PTC as a biomarker or tumor marker, we investigated the presence of aberrant $X A F 1$ transcripts in cancer cell lines and in the peripheral blood of patients with gastric cancer using qRT-PCR. The RT-PCR analysis and DNA sequencing revealed that a novel splice variant of $X A F 1$ was expressed in gastric, pancreatic, colorectal, and breast cancer cell lines. This splice variant harboring PTC accumulated in NMD-suppressed cells. Furthermore, the $X A F 1$ variant in peripheral blood containing CTCs obtained from patients with gastric cancer was significantly upregulated relative to samples from healthy volunteers. These findings suggest that the novel $X A F 1$ variant identified in this study is a potential blood biomarker.

\section{Materials and methods}

Patients and specimens

From April 2010 to August 2012, PAXgene (PreAnalytiX; Hombrechtikon, Switzerland) was used to collect peripheral blood samples $(2.5 \mathrm{ml})$ from 96 patients [65 men, 31 women; median age, 67 (30-85) years] with gastric cancer at Shizuoka Cancer Center Hospital and from 22 healthy volunteers [16 men, 6 women; median age, 36 (26-70) years] who were coworkers at the hospital. Informed consent was obtained from all patients, and the Institutional Review Board at Shizuoka Cancer Center approved all aspects of this study.

\section{Cell cultures, RNA sample preparation, and RT-PCR}

The cell lines used in this study are listed in Table S1 in the Supplementary Material. cDNA for different splice variants of XAFl was screened using the intron-spanning exonic primers listed in Table S2 in the Supplementary Material. The methods are described in detail in the Supplementary Material.

DNA sequencing analysis

To determine the sequence of the novel XAF1 transcript, rapid amplification of cDNA ends (RACE) was conducted 
using the GeneRacer Kit (Life Technologies, Carlsbad, CA, USA) according to the manufacturer's instructions. The primer sequences are shown in Table S2. The methods used for DNA sequencing analysis are provided in detail in the Supplementary Material.

\section{Quantitative real-time PCR}

Quantitative real-time PCR (qRT-PCR) was performed using the SYBR Green dye technique and the ABI PRISM 7900HT Fast Real-Time PCR System (Life Technologies). The methods and the validation results are described in detail in the Supplementary Material.

NMD inhibition assay with caffeine

The method for inhibiting NMD was previously described in detail [37]. Briefly, cells were seeded in two culture plates, and caffeine $(10 \mathrm{mM})$ was added to one plate. Following $4 \mathrm{~h}$ of incubation, the medium was removed from both plates and the cells were washed twice with phosphate-buffered saline. Both actinomycin D (actD, $2 \mathrm{mg} / \mathrm{ml})$ and caffeine $(10 \mathrm{mM})$ were added to one plate (pretreated with caffeine), and actD alone was added to the other plate. After a further $4 \mathrm{~h}$ incubation, total RNA was obtained from both plates (see details in the Supplementary Material).

\section{Statistical analysis}

The coefficient of determination in the qRT-PCR standard curve was derived by Pearson's correlation analysis. XAF1 and survivin (Baculoviral IAP Repeat Containing 5, BIRC5) expression levels in gastric cancer patients and healthy volunteers were depicted as box plots containing outliers and extreme outliers. The inner and outer fences for the plotting of the outliers were calculated using the $1.5 \times$ interquartile range (IQR) and $3.0 \times \mathrm{IQR}$, respectively. Because of the difference in sample size between patients and volunteers, the $p$ value of the qRT-PCR analysis was calculated based on Welch's $t$ test. Receiver operating characteristic (ROC) curves were computed using the $\mathrm{R}$ software and the associated pROC package [38]. The area under the curve (AUC) and optimal threshold (cutoff value), i.e., the point closest to the top left in the plot, were calculated in R. The $95 \%$ confidence interval (CI) was computed to assess the variability of the measure using 10,000 bootstrap replicates [39]. Gene expression and clinicopathological characteristics according to the Japanese Classification of Gastric Carcinoma, 14th edition, were compared using Fisher's exact test. Significance was defined as $p<0.05$.

\section{Results}

Identification of novel $X A F 1$ exons

Several splice variants of $X A F 1$ are known to be expressed in cancer cell lines [11-14]. To analyze the variants, we initially characterized the expression pattern of $X A F 1$ in the highly metastatic gastric cancer cell MKN45P (Fig. 1a). RT-PCR was conducted using previously reported primers [12] and newly designed primers to detect known transcripts and novel variants, respectively. Electrophoresis of the RT-PCR amplicons revealed that an unknown transcript candidate $(X A F 1 F)$ was coexpressed with XAF1A and $X A F 1 C$ in MKN45P. PCR-based cloning and DNA sequencing analysis using RACE subsequently showed that $X A F 1 F$ was a novel splice variant (Fig. 1b). Specifically, this variant lacked exon 5 and possessed a unique exon 3 that contained exon 3-ext derived from the intronic region, resulting in seven exons. The sequence of the novel exonic region had a stop codon that was regarded as a PTC.

\section{$X A F 1 F$ expression in cancer cell lines}

XAF1 transcripts are downregulated or absent in colorectal cancer cells [12]. We thus investigated the expression profile of $X A F 1 F$ transcripts in gastrointestinal (colorectal, gastric, and pancreatic) and breast cancer cell lines using RT-PCR analysis (Fig. 2). The XAF1F transcript was expressed in 20 cell lines $(20 / 45,44 \%)$ and also coexisted with other XAF1 transcripts $(X A F 1 A / C)$ in 20 cell lines $(20 / 20,100 \%)$. However, 10 of 32 XAFlAexpressing cell lines did not express the XAF1F transcript $(10 / 32,31 \%)$. These results suggest that although XAF1F harboring PTC was often degraded by the NMD pathway, this transcript was coexpressed with other XAFls in many cancer cells.

mRNA expression of NMD-related genes

Although $X A F 1 F$ possessed a PTC in its mature mRNA sequence, it was expressed in 20 cancer cell lines. Among them, comparison of two pairs of cell lines (MKN45P vs. MKN45 and KP-3L vs. KP-3) in a metastatic model obtained by xenografting (for details, see Table S1) revealed that XAF1 expression, including that of $X A F 1 F$, in highly metastatic cells was higher than that in the parental cells (Fig. 2 and S2 in the Supplementary Material). To investigate NMD activity in variantexpressing cells, qRT-PCR for NMD-related genes was performed using these pairs (Fig. 3a). In MKN45P cells, the NMD target transcripts ATF3 $[16,40]$ and $M A F F$ 
a

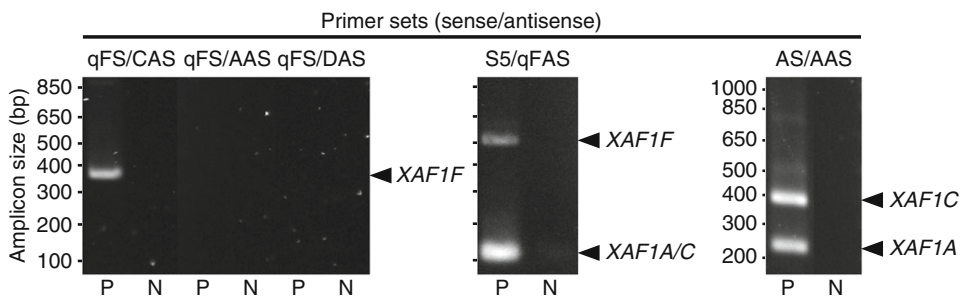

b

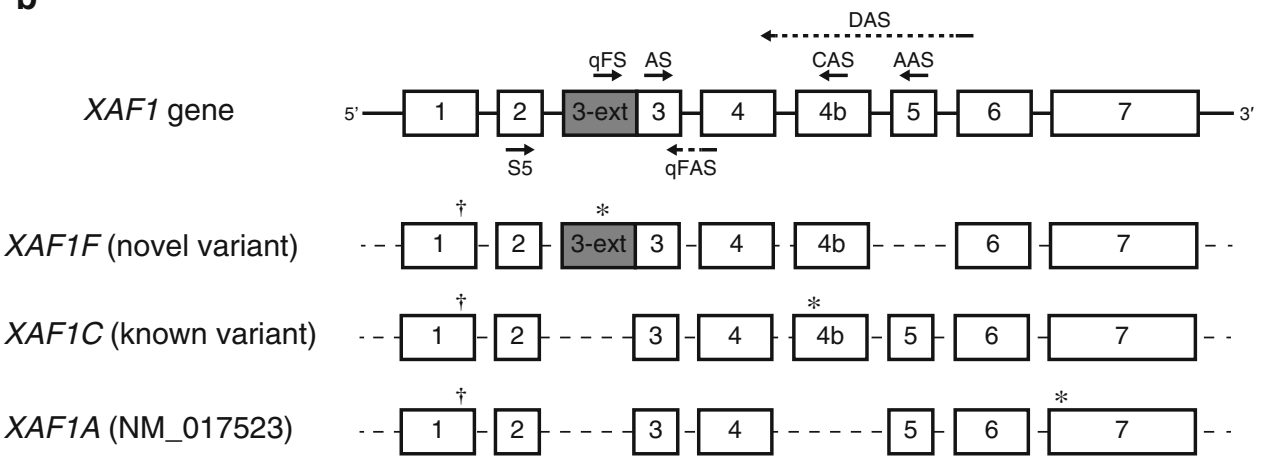

Fig. 1 Identification of a novel XIAP-associated factor 1 (XAF1) transcript. a Detection of $X A F 1$ transcripts expressed in a gastric cancer cell line (MKN45P). The novel transcript $(X A F 1 F)$ was detected by RT-PCR using the primer sets qFS/CAS and S5/qFAS (sense/antisense). $P$ and $N$ under the individual lanes indicate the positive control (RT-PCR with MKN45P template) and negative control (without template), respectively. b Schematic representation of the exon structures of the $X A F 1$ gene and transcripts identified in this study. The gray boxes show a novel exonic region extending from known exon 3 in the $X A F 1$ gene, resulting in 8 exons. The primer positions for RT-PCR are indicated by arrows. Primers qFAS and DAS are designed to step over the intron between exons 3 and 4 and exons $4 \mathrm{~b}$ and 5, respectively. The dagger and asterisk symbols indicate the locations of the start and stop codon, respectively
Fig. 2 Expression profile of $X A F 1$ transcripts in gastric, colorectal, pancreatic, and breast cancer cell lines. The transcripts of $X A F 1 F$ and $X A F 1 A / C$ were detected by RTPCR using specific primer sets qFS/CAS and AS/AAS (sense/ antisense), respectively. RTPCR for beta-actin $(A C T B)$ was conducted as a positive control experiment. Agarose gels were used at a concentration of $2 \%$
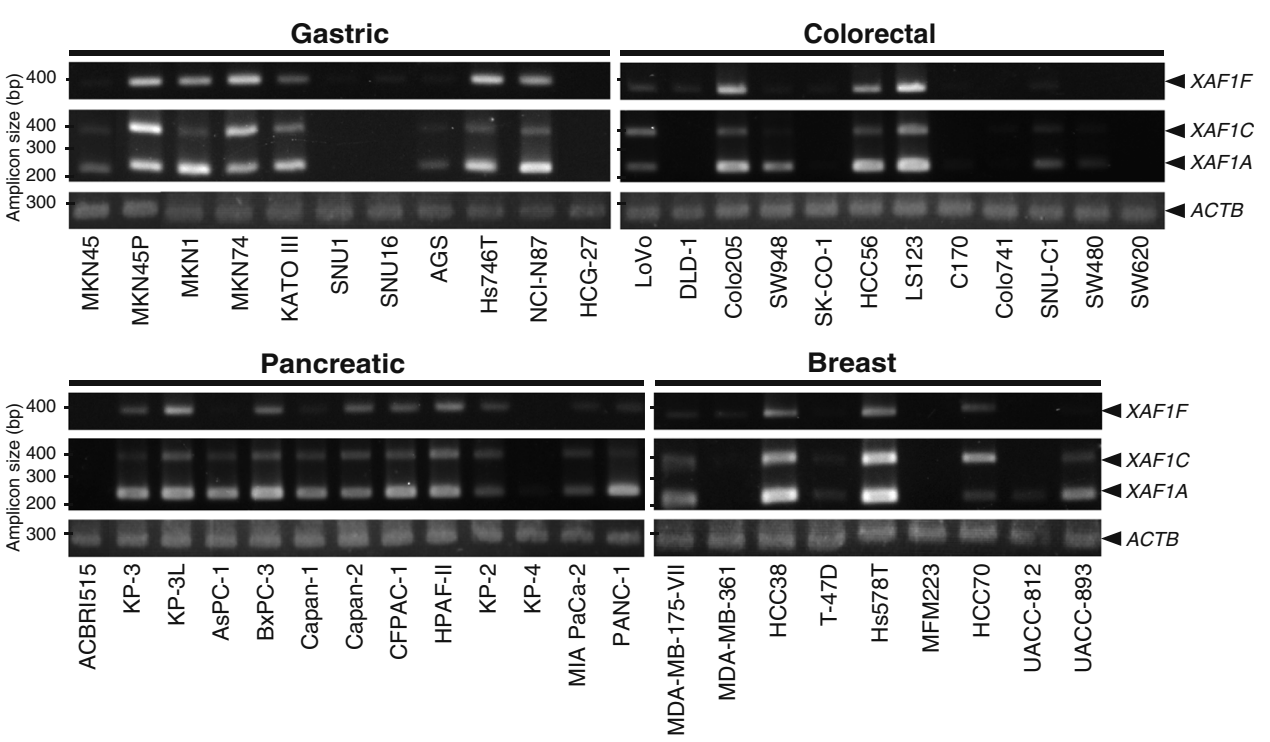

[41] were upregulated but the NMD factor UPF1 [16, 41] was downregulated, indicating that the NMD pathway was inhibited. In KP-3L cells, mRNA expression of $A T F 3, G A D D 45 B$ [16], and UPF1 was significantly increased.
XAF1 expression in NMD-inhibited cells

To determine whether the transcript was degraded through the NMD pathway, we performed an NMD inhibition assay using caffeine and actD (Fig. 3b). The combination of 
a

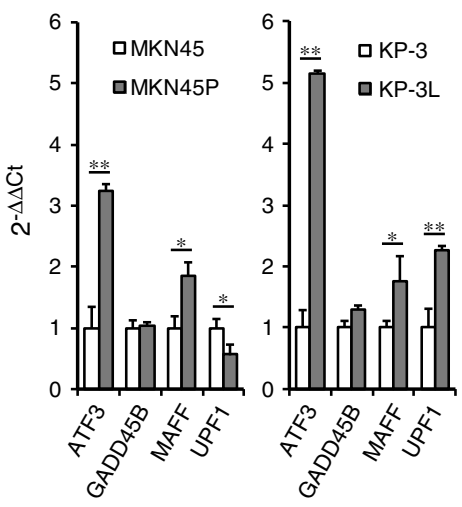

b

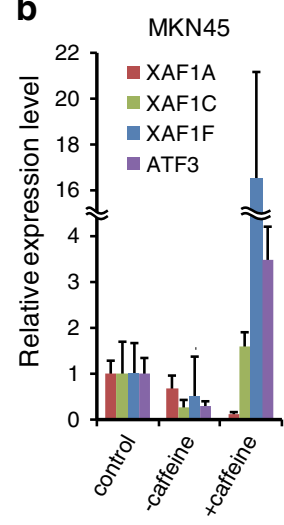

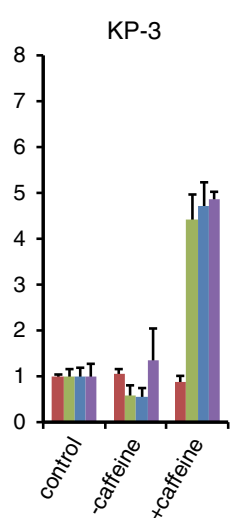

KP-3L

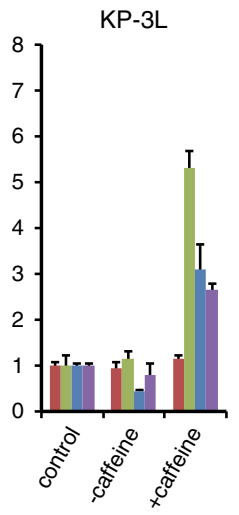

Fig. 3 Expression analysis of nonsense-mediated mRNA decay (NMD)-related and $X A F 1$ transcripts in pairs of cell lines used as a metastatic model. a Comparison of NMD-related gene expression between highly metastatic cells and their parental cells. The pairs of gastric cells (MKN45 vs. MKN45P) and pancreatic cells (KP-3 vs. $\mathrm{KP}-3 \mathrm{~L})$ are shown in left and right panels, respectively. The expression levels in the parental cells (MKN45 or KP-3) are shown as 1.0. SD was calculated from the relative expression level of triplicates. Statistical significance $(* p<0.05$ and $* * p<0.01)$ was evaluated by Student's $t$ test. b Expression levels of XAF1 transcripts in NMD-inhibited cancer cells. NMD was inhibited by caffeine and actD treatment (+caffeine). Treatment with actD alone (-caffeine) was used to observe RNA stability after blockade of transcription. Cancer cells that were not treated with reagents are presented as the control. The expression levels of XAF1A and XAF1C were estimated based on previous reports [12]. qRT-PCR of $A T F 3$ was conducted to determine NMD inhibition

splice variants were upregulated in the peripheral blood of gastric cancer patients.

mRNA expression of NMD-related genes in peripheral blood containing CTCs

qRT-PCR performed using peripheral blood collected with PAXgene can detect transcripts in CTCs [33, 34]. Recently, several research groups reported that CTC-derived survivin (BIRC5) was frequently detected in the peripheral blood of gastrointestinal cancer patients and that the mRNA expression level of this gene is useful as a prognostic factor [36, 44-47]. Therefore, to isolate CTC-positive samples, we adopted the BIRC5 gene as a marker and performed a qRT-PCR assay for the quantification of BIRC5 and $X A F 1 F$ transcripts (Fig. S4 in the Supplementary Material). The prediction performance for CTC detection was evaluated using a ROC curve (Fig. 5). The AUC and cutoff values were 0.8299 (95\% CI, 0.7311-0.9048) and 180.75 (sensitivity, $82.99 \%$; specificity, $77.27 \%$ ), respectively. These data indicate that BIRC5 expression, as measured by our qRT-PCR assay, is an efficient predictor for CTCs in the peripheral blood of gastric cancer patients. Using the calculated cutoff value, 79 CTC-positive samples were identified from 96 patients with gastric cancer.

To investigate the relationship between $X A F 1$ splice variants and the NMD target gene ATF3 in the CTCpositive population, we also performed a ROC curve analysis for $X A F 1 F$ and $X A F 1 C$ (Fig. S5 in the Supplementary Material). Although the AUC of the variants was lower to negligible correlation, suggesting that the expression was independent of age. These results indicate that $X A F 1$ 


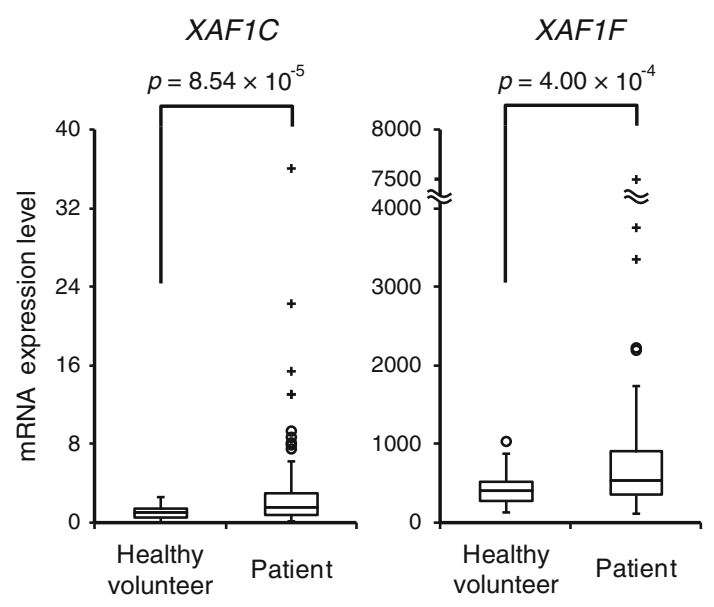

Fig. 4 Quantification of $X A F 1 F$ and $X A F 1 C$ transcripts in peripheral blood. $X A F 1 F$ and $X A F 1 C$ expression in gastric cancer patients ( $n=96)$ and healthy volunteers $(n=22)$ was quantified using the qRT-PCR assay described in Figure S1 in the Supplementary Material. The normal and extreme outliers are indicated by circle and plus symbols, respectively

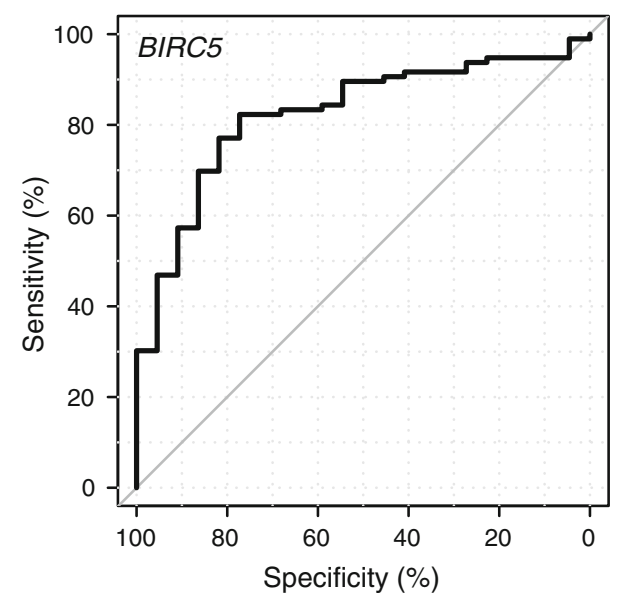

Fig. 5 ROC curve of BIRC5 expression in peripheral blood. The black solid line indicates the curve for BIRC5 in all peripheral blood samples $(n=118)$

than that of $B I R C 5$, the cutoff values were determined from the ROC curve. Table 1 shows $A T F 3$ expression in $X A F 1 C / F$-positive and $X A F 1 C / F$-negative populations distinguished by these values. The $X A F 1 F$ - and $X A F 1 C$ positive patients accounted for $62.0 \%(49 / 79)$ and $45.6 \%$ (36/79), respectively, of the patients with CTCs. The population that was positive for both variants expressed the ATF3 transcript. The expression level of ATF3 in the positive patients was higher than that in the negative patients.

To further evaluate the differences in the patients with CTCs in the $X A F 1 C / F$-positive and $X A F I C / F$-negative populations, we correlated clinicopathological characteristics with the expression level of the splice variants
Table 1 mRNA expression level of XAF1 variants and $A T F 3$ in gastric cancer patients

\begin{tabular}{|c|c|c|c|}
\hline & \multirow{2}{*}{$\begin{array}{l}\text { Sample } \\
\text { number }\end{array}$} & \multicolumn{2}{|l|}{ ATF3 } \\
\hline & & $\begin{array}{l}\text { Expression level } \\
(\text { mean } \pm \text { SD) }\end{array}$ & $p$ value \\
\hline \multicolumn{4}{|l|}{ In all patients } \\
\hline BIRC5+ & 79 & $1.06 \pm 0.51$ & NS \\
\hline BIRC5- & 17 & $0.87 \pm 0.47$ & \\
\hline \multicolumn{4}{|c|}{ In $B I R C 5$-positive patients } \\
\hline$X A F 1 F+$ & 49 & $1.15 \pm 0.54$ & 0.032 \\
\hline$X A F 1 F-$ & 30 & $0.92 \pm 0.42$ & \\
\hline$X A F 1 C+$ & 36 & $1.27 \pm 0.61$ & 0.0013 \\
\hline$X A F 1 C-$ & 43 & $0.89 \pm 0.31$ & \\
\hline$X A F 1 F / C+$ & 26 & $1.33 \pm 0.62$ & 0.0040 \\
\hline$X A F 1 F / C-$ & 53 & $0.93 \pm 0.38$ & \\
\hline
\end{tabular}

Test used: Welch's $t$ test

$N S$ not significant

(Table 2). Among tumors invading into the subserosa (SS) or further, $74 \%$ (29/39) expressed the XAFIF transcript, whereas $50 \%(20 / 40)$ of tumors invading the muscularis propria (MP) were XAFIF negative $(p=0.0368)$. Comparison of patients with $X A F 1 F$ expression demonstrated that $59 \%(29 / 49)$ of the tumors in those patients invaded as far as the SS or further. Among patients with venous invasion, $80 \%(32 / 40)$ were $X A F 1 F$ positive, whereas $56 \%(22 / 39)$ of patients without venous invasion did not show expression of XAFIF $(p=0.0011)$. In the XAF1Fpositive population, $65 \%(32 / 49)$ of the patients had evidence of venous invasion. Furthermore, $76 \%(32 / 42)$ of patients with lymph node metastasis were $X A F 1 F$ positive $(p=0.0101)$. To investigate the relationship between this metastasis and lymphatic invasion, we further analyzed the frequency of invasion in $X A F 1 F$-positive patients with lymph node metastasis. Although this splice variant was not correlated with lymphatic invasion in 79 patients with CTCs, 27 of 32 patients with metastasis $(\mathrm{N} 1,2,3)$ had lymphatic invasion (84\%), whereas $82 \%$ of patients without metastasis (N0) had no lymphatic invasion (14/17, $p=7.528 \times 10^{-6}$ in Fisher's exact test). This result indicates that the lymph node metastasis associated with $X A F 1 F$-expressing CTCs accompanies lymphatic invasion. These CTCs may therefore easily metastasize to a lymph node rather than a lymphatic vessel.

\section{Discussion}

Alternative splicing allows a single gene to generate multiple mRNAs that can be translated into diverse proteins 
Table 2 Expression of XAF1F and XAF1C in clinicopathological characteristics of circulating tumor cell (CTC)-containing patients

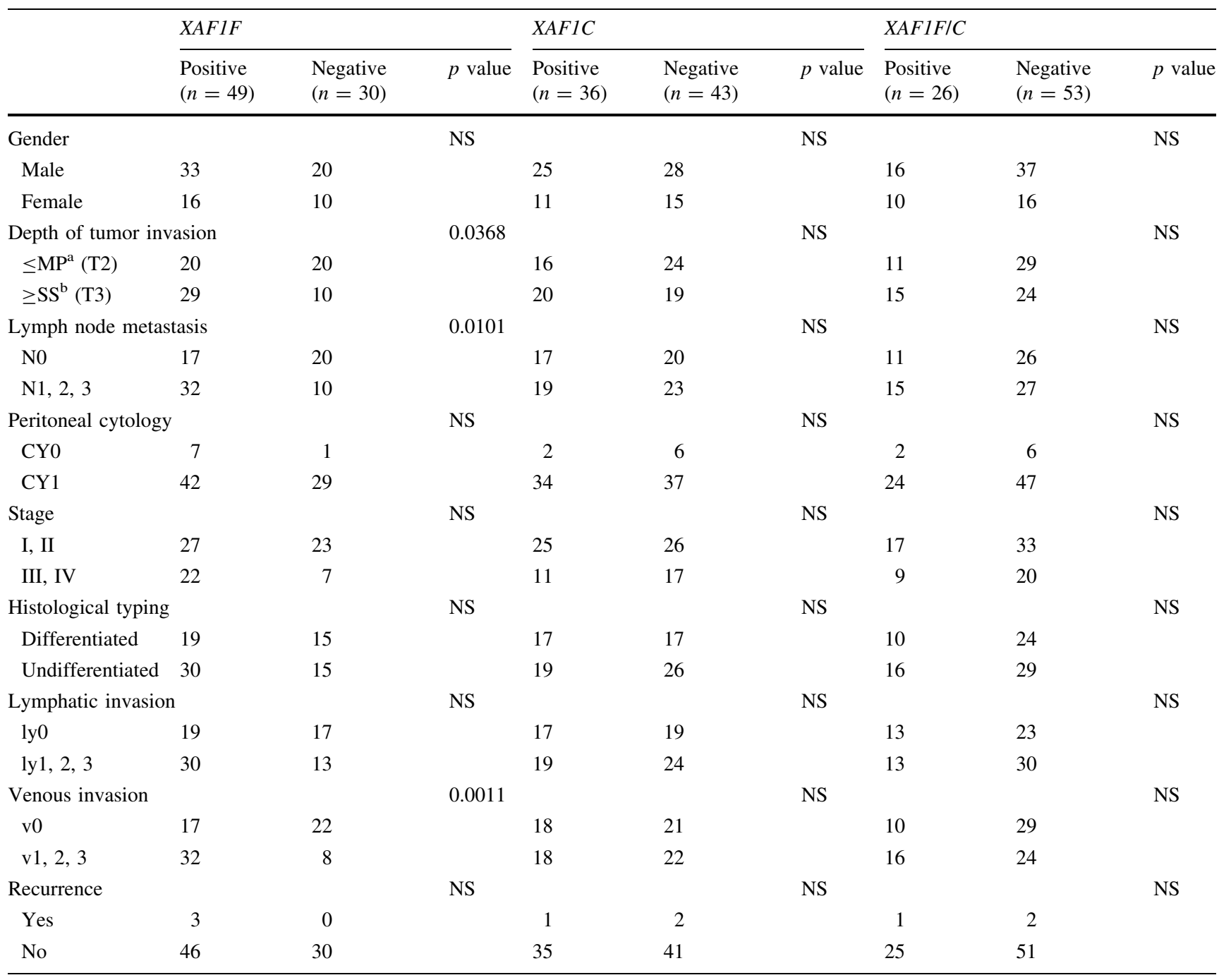

Test used: Fisher's exact test

NS not significant

${ }^{\text {a }}$ Muscularis propria

${ }^{\mathrm{b}}$ Subserosa

[48]. Many transcripts have been predicted by in silico approaches and registered in public databases (e.g., Ensembl, http://www.ensembl.org) as candidate splice variants [49, 50]. Recently, Furuta et al. [51, 52] and our research group independently found that aberrant alternative splicing in cancer cells results in the insertion of intronic regions as extended exons, which results in the generation of new splice variants. Thus, it is important to explore the intronic regions of target genes to find novel splice variants, and here, we investigated the exons and introns of $X A F 1$ simultaneously.

In agreement with previous reports [2, 12], XAF1 was downregulated in more than half the cell lines tested. However, the $X A F 1 F$ transcript harboring a PTC was not often coexpressed with other $X A F 1$ transcripts. Comparative analysis of metastatic models obtained by xenografting (MKN45P vs. MKN45 and KP-3L vs. KP-3) revealed that the $X A F 1 F$ and NMD target genes were upregulated in MKN45P and KP-3L cells. These data suggest that the NMD pathway in $X A F 1 F$-expressing cells with metastatic potential is suppressed relative to the parent cells. Recent studies have shown that tumor growth and metastasis are facilitated by NMD inhibition [53, 54]. The suppression of the NMD pathway in MKN45P and KP-3L cells may therefore be associated with cancer metastasis.

We found that the $X A F 1 F$ transcript harboring PTC was upregulated in NMD-suppressed cancer cells and presumably subjected to NMD. To elucidate the degradation of the 
$X A F 1 F$ transcript through the NMD pathway, an NMD inhibition assay was performed. The NMD factor UPF1 and NMD target genes (Fig. 3a) were significantly upregulated in KP-3L cells, which raises the possibility that NMD inhibition in $X A F 1 F$-expressed cells is regulated by other NMD factors. Therefore, we used caffeine as an NMD inhibitor [37] rather than depletion of UPF1 by RNAi [16]. In cancer cells treated with only actD, which blocks transcription, XAFlF expression was decreased, indicating that this transcript is especially unstable in cells with active NMD. In contrast, $X A F 1 F$ accumulated in cells in which NMD was inhibited with caffeine. These results suggest that the PTC-harboring XAF1F is degraded through the NMD pathway. Therefore, we concluded that expression analysis of this splice variant is valuable to evaluate NMD inhibition in cancer cells.

Splice variants of XAF1 have been found to be significantly upregulated in the peripheral blood of gastric cancer patients. Furthermore, survivin, which is considered to be expressed in CTCs, is also detected in many patients. Therefore, $X A F 1$ variants are likely to be derived from CTCs. However, use of the qRT-PCR assay and PAXgene cannot eliminate the influence of circulating cell-free RNA [55] (including mRNA in microvesicles or exosomes [56, 57] ) and lymphocytes or other nucleated cells because of the stabilization of whole RNA in the peripheral blood. Further studies should thus investigate the expression of $X A F 1$ variants in CTCs isolated using immunomagnetic separation systems (such as CellSearch). However, Chi et al. reported that $X A F 1$ transcripts are significantly downregulated in gastric and colorectal tumors [2, 12]. If the upregulated $X A F 1$ transcripts that we observed are derived from CTCs, then the expression levels of these transcripts would thus be inconsistent between the tumor tissue and CTCs. Several reports described such a difference in the gene expression profiles of primary tumors and CTCs $[58,59]$. This discrepancy should be also examined using isolated CTCs in further studies.

We further attempted to discriminate survivin-positive patients using the cutoff value calculated from the ROC curve to identify the population who had CTCs. Among the patients, $82 \%$ (79/96) were categorized as survivinexpressing CTC-positive patients, which was similar to the discriminative performance in a previous study using RTPCR ELISA [35]. Furthermore, the $X A F 1 F / C$-positive population accounted for approximately half the CTCpositive patients and showed significant expression of the ATF3 transcript. These results suggest that the NMD pathway is often suppressed in peripheral blood containing survivin-expressing CTCs derived from gastric cancer. Recently, several research groups have reported that heterogeneity may lead to differences in protein expression or cellular adhesion in CTCs [60-62]. The NMD-suppressed population identified in this study may also contribute to the heterogeneity of CTCs.

XAF1 in cancer cells acts as a tumor suppressor because of its pro-apoptotic function (Fig. 6) [13]. XAF1 expression in various cancer cell types was found to be transcriptionally inactivated by the methylation of $\mathrm{CpG}$ sites in the promoter region $[9,10]$. Heat shock factor (HSF) 1 and p53 can also negatively regulate the $X A F 1$ gene via the binding of these binding elements [63, 64]. Several studies have demonstrated that $X A F 1$ is upregulated by interferon (IFN), resulting in the sensitization of cells to apoptosis [65-69]. To our knowledge, this study is the first to show that $X A F 1 F$ is generated by aberrant pre-mRNA processing through NMD inhibition, which is often induced by cellular stress in the tumor microenvironment [17-19]. The XAF1Fexpressing cells with inhibition of NMD may be stressed by external stimuli or have an amplified cellular stress response. Our qRT-PCR assay revealed that the NMD pathway tends to be inhibited in some CTCs from gastric cancer. Several studies have demonstrated that CTCs exposed to blood flow undergo physiological shear stress, leading to a change in the gene expression pattern $[70,71]$. These findings suggest that XAF1F-expressing CTCs with inhibition of NMD may be strongly stressed by the external environment in comparison with the $X A F I F$-negative population or that they may be highly sensitive to stress.

Recent studies detected CTCs from gastric or hepatocellular cancer in patients with vascular invasion [72, 73], whereas CTCs derived from head and neck cancer were associated with lymph node metastasis [74, 75], indicating that these characteristics depend on the primary tumor. In our study, the mRNA expression level of XAFIF in CTCs was significantly correlated with venous invasion, lymph node metastasis, and tumor invasion that reached the SS. Additionally, the lymph node metastasis associated with $X A F 1 F$-expressing CTCs accompanied lymphatic invasion. Metastatic cells in which the EMT has occurred have been shown to penetrate local tissue and blood or lymphatic vessels [76]. These findings raise the possibility that $X A F 1 F$-positive CTCs in gastric cancer have the characteristics of EMT. In several cancer cell types, the EMT is closely related with RNA splicing [28] or NMD inhibition that can generate aberrant transcripts [29]. Therefore, CTCs expressing splice variants via NMD suppression may have been phenotypically converted by the EMT. At the minimum, these findings suggest that expression of both $X A F 1 F$ and survivin in the peripheral blood of gastric cancer patients is a predictor of venous invasion, lymph node metastasis, and depth of tumor invasion.

A significant correlation was found between $X A F 1 F$ in CTCs and the depth of tumor invasion/lymph node metastasis, whereas XAF1F expression tended to be associated with the stage of gastric cancer $(p=0.05985$ in 
Fig. 6 Key roles of XAF1 in cancer cells. The transcription of the XAF1 gene is under the control of negative and positive regulatory elements. NMD inhibition promotes accumulation of XAF1F. Gray arrows indicate the upregulation or downregulation of the $X A F 1$ transcripts. HSF and XIAP represent heat shock factor 1 and XIAP, respectively

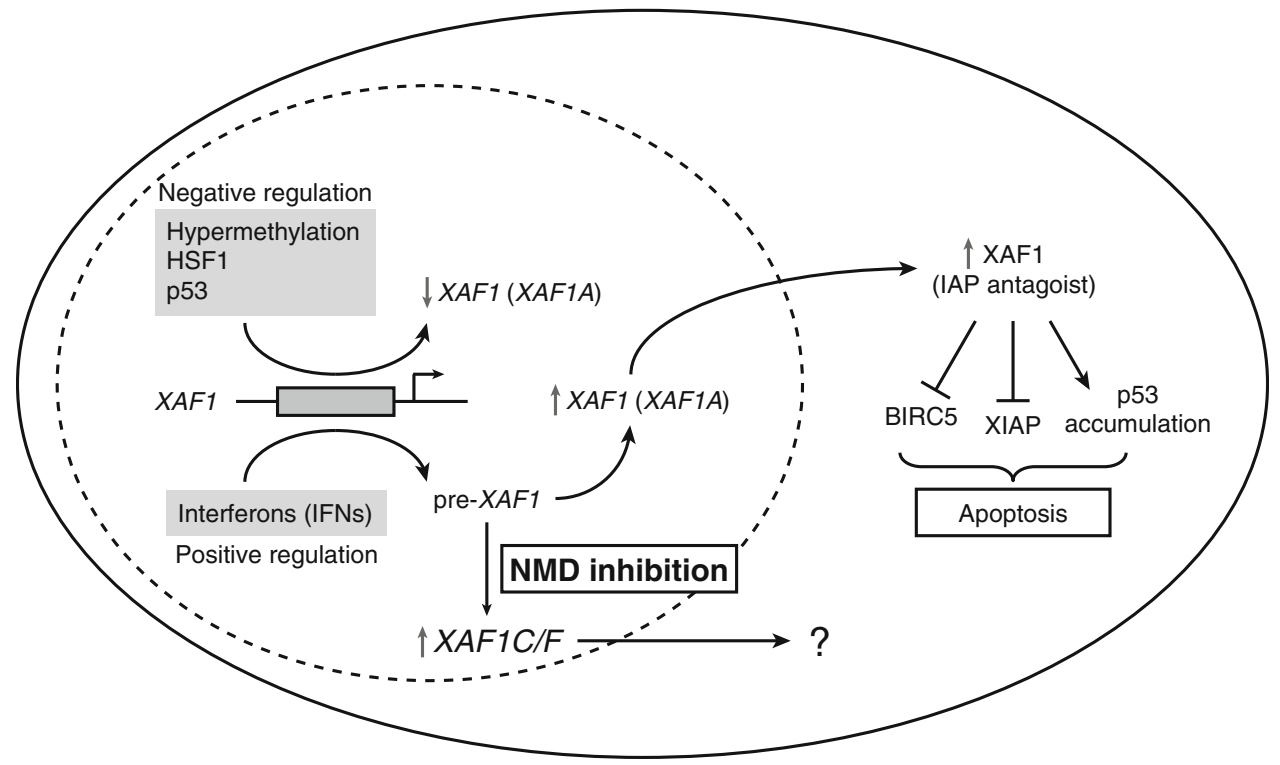

Fisher's exact test). In stage I and II, $67 \%(2 / 3)$ and $90 \%$ $(9 / 10)$ of patients with lymph node metastasis were categorized as $X A F 1 F$ positive, respectively. These results suggest that $X A F 1 F$ expression in CTCs strongly reflects lymph node metastasis in stage I and II, leading to a weak association between $X A F 1 F$ expression and tumor stage. Additionally, $X A F 1 F$ expression in CTCs was not associated with recurrence, although CTCs are useful as a prognostic factor [36, 44-47]. In further studies, this discrepancy should be also examined using long-term followup data of CTCs isolated from patients to clarify the origin of the XAF1 variant.

In conclusion, the present study identified a novel splice variant of $X A F 1$ in gastric, pancreatic, colorectal, and breast cancer cells. This variant was regulated through the NMD pathway and accumulated in NMD-suppressed metastatic cancer cells. To our knowledge, this is the first study to clearly detect $X A F 1$ variants in peripheral blood containing CTCs derived from gastric cancer. The expression of these variants was significantly higher than that in healthy volunteers. Furthermore, in part of the CTCpositive population, the NMD pathway was suppressed. These findings suggest that $X A F 1$ variants accumulate by NMD inhibition in the peripheral blood of gastric cancer patients and may indicate heterogeneity of CTCs.

Acknowledgments This research was supported by a Grant-inCooperation of the Regional Innovation Cluster Program 2010 and Japan Society for the Promotion of Science KAKENHI 23701092 and 25710013. The authors thank Yuko Watanabe, Kaori Kanto, and Tomomi Ide for their technical assistance, Keita Mori for statistical advice, and Dr. Takashi Sugino for comments on the clinicopathological data.

Conflict of interest The authors have no conflict of interest.

\section{References}

1. Liston P, Fong WG, Kelly NL, et al. Identification of XAF1 as an antagonist of XIAP anti-caspase activity. Nat Cell Biol. 2001;3(2):128-33.

2. Byun DS, Cho K, Ryu BK, et al. Hypermethylation of XIAPassociated factor 1, a putative tumor suppressor gene from the 17 p13.2 locus, in human gastric adenocarcinomas. Cancer Res. 2003;63(21):7068-75.

3. Arora V, Cheung HH, Plenchette S, et al. Degradation of survivin by the X-linked inhibitor of apoptosis (XIAP)-XAF1 complex. J Biol Chem. 2007;282(36):26202-9.

4. Tu SP, Liston P, Cui JT, et al. Restoration of XAF1 expression induces apoptosis and inhibits tumor growth in gastric cancer. Int J Cancer. 2009;125(3):688-97.

5. Huang J, Yao WY, Zhu Q, et al. XAF1 as a prognostic biomarker and therapeutic target in pancreatic cancer. Cancer Sci. 2010;101(2):559-67.

6. Sun PH, Zhu LM, Qiao MM, et al. The XAF1 tumor suppressor induces autophagic cell death via upregulation of Beclin-1 and inhibition of Akt pathway. Cancer Lett. 2011;310(2):170-80.

7. Zou B, Chim CS, Pang R, et al. XIAP-associated factor 1 (XAF1), a novel target of $\mathrm{p} 53$, enhances $\mathrm{p} 53$-mediated apoptosis via posttranslational modification. Mol Carcinog. 2012;51(5):422-32.

8. Fong WG, Liston P, Rajcan-Separovic E, et al. Expression and genetic analysis of XIAP-associated factor 1 (XAF1) in cancer cell lines. Genomics. 2000;70(1):113-22.

9. Zou B, Chim CS, Zeng H, et al. Correlation between the singlesite $\mathrm{CpG}$ methylation and expression silencing of the XAF1 gene in human gastric and colon cancers. Gastroenterology. 2006;131(6): 1835-43.

10. Lee MG, Huh JS, Chung SK, et al. Promoter CpG hypermethylation and downregulation of XAF1 expression in human urogenital malignancies: implication for attenuated p53 response to apoptotic stresses. Oncogene. 2006;25(42):5807-22.

11. Yin W, Cheepala S, Clifford JL. Identification of a novel splice variant of X-linked inhibitor of apoptosis-associated factor 1 . Biochem Biophys Res Commun. 2006;339(4):1148-54.

12. Chung SK, Lee MG, Ryu BK, et al. Frequent alteration of XAF1 in human colorectal cancers: implication for tumor cell resistance to apoptotic stresses. Gastroenterology. 2007;132(7):2459-77. 
13. Plenchette $\mathrm{S}$, Cheung HH, Fong WG, et al. The role of XAF1 in cancer. Curr Opin Invest Drugs. 2007;8(6):469-76.

14. Fang X, Liu Z, Fan Y, et al. Switch to full-length of XAF1 mRNA expression in prostate cancer cells by the DNA methylation inhibitor. Int J Cancer. 2006;118(10):2485-9.

15. Maquat LE. Nonsense-mediated mRNA decay. Curr Biol. 2002;12(6):R196-7.

16. Mendell JT, Sharifi NA, Meyers JL, et al. Nonsense surveillance regulates expression of diverse classes of mammalian transcripts and mutes genomic noise. Nat Genet. 2004;36(10):1073-8.

17. Gardner LB. Nonsense-mediated RNA decay regulation by cellular stress: implications for tumorigenesis. Mol Cancer Res. 2010;8(3):295-308.

18. Gardner LB. Hypoxic inhibition of nonsense-mediated RNA decay regulates gene expression and the integrated stress response. Mol Cell Biol. 2008;28(11):3729-41.

19. Wang D, Zavadil J, Martin L, et al. Inhibition of nonsensemediated RNA decay by the tumor microenvironment promotes tumorigenesis. Mol Cell Biol. 2011;31(17):3670-80.

20. Tani H, Torimura M, Akimitsu N. The RNA degradation pathway regulates the function of GAS5 a non-coding RNA in mammalian cells. PLoS One. 2013;8(1):e55684.

21. Wang S, Zheng G, Cheng B, et al. Circulating tumor cells (CTCs) detected by RT-PCR and its prognostic role in gastric cancer: a meta-analysis of published literature. PLoS One. 2014;9(6):e99259.

22. Powell AA, Talasaz AH, Zhang H, et al. Single cell profiling of circulating tumor cells: transcriptional heterogeneity and diversity from breast cancer cell lines. PLoS One. 2012;7(5):e33788.

23. Neuert G, Munsky B, Tan RZ, et al. Systematic identification of signal-activated stochastic gene regulation. Science. 2013;339(6119):584-7.

24. Zhang ZY, Ge HY. Micrometastasis in gastric cancer. Cancer Lett. 2013;336(1):34-45.

25. Savagner $P$, Valles AM, Jouanneau J, et al. Alternative splicing in fibroblast growth factor receptor 2 is associated with induced epithelial-mesenchymal transition in rat bladder carcinoma cells. Mol Biol Cell. 1994;5(8):851-62.

26. Pino MS, Balsamo M, Di Modugno F, et al. Human Mena+11a isoform serves as a marker of epithelial phenotype and sensitivity to epidermal growth factor receptor inhibition in human pancreatic cancer cell lines. Clin Cancer Res. 2008;14(15):4943-50.

27. Warzecha CC, Sato TK, Nabet B, et al. ESRP1 and ESRP2 are epithelial cell-type-specific regulators of FGFR2 splicing. Mol Cell. 2009;33(5):591-601.

28. Shapiro IM, Cheng AW, Flytzanis NC, et al. An EMT-driven alternative splicing program occurs in human breast cancer and modulates cellular phenotype. PLoS Genet. 2011;7(8):e1002218.

29. Valacca C, Bonomi S, Buratti E, et al. Sam68 regulates EMT through alternative splicing-activated nonsense-mediated mRNA decay of the SF2/ASF proto-oncogene. J Cell Biol. 2010;191(1):87-99.

30. Fujita Y, Terashima M, Hoshino Y, et al. Detection of cancer cells disseminated in bone marrow using real-time quantitative RT-PCR of CEA, CK19, and CK20 mRNA in patients with gastric cancer. Gastric Cancer. 2006;9(4):308-14.

31. Huang P, Wang J, Guo Y, et al. Molecular detection of disseminated tumor cells in the peripheral blood in patients with gastrointestinal cancer. J Cancer Res Clin Oncol. 2003;129(3):192-8.

32. Ishigami S, Sakamoto A, Uenosono Y, et al. Carcinoembryonic antigen messenger RNA expression in blood can predict relapse in gastric cancer. J Surg Res. 2008;148(2):205-9.

33. Helo P, Cronin AM, Danila DC, et al. Circulating prostate tumor cells detected by reverse transcription-PCR in men with localized or castration-refractory prostate cancer: concordance with Cell Search assay and association with bone metastases and with survival. Clin Chem. 2009;55(4):765-73.
34. Danila DC, Anand A, Schultz N, et al. Analytic and clinical validation of a prostate cancer-enhanced messenger RNA detection assay in whole blood as a prognostic biomarker for survival. Eur Urol 2014;65(6):1191-7

35. Cao W, Yang W, Li H, et al. Using detection of survivinexpressing circulating tumor cells in peripheral blood to predict tumor recurrence following curative resection of gastric cancer. J Surg Oncol. 2011;103(2):110-5.

36. Cao M, Yie SM, Wu SM, et al. Detection of survivin-expressing circulating cancer cells in the peripheral blood of patients with esophageal squamous cell carcinoma and its clinical significance. Clin Exp Metastasis. 2009;26(7):751-8.

37. Ivanov I, Lo KC, Hawthorn L, et al. Identifying candidate colon cancer tumor suppressor genes using inhibition of nonsensemediated mRNA decay in colon cancer cells. Oncogene. 2007;26(20):2873-84.

38. Robin X, Turck N, Hainard A, et al. pROC: an open-source package for $\mathrm{R}$ and $\mathrm{S}+$ to analyze and compare ROC curves. BMC Bioinform. 2011;12:77.

39. Xia J, Broadhurst DI, Wilson M, et al. Translational biomarker discovery in clinical metabolomics: an introductory tutorial. Metabolomics. 2013;9(2):280-99.

40. Huang L, Lou CH, Chan W, et al. RNA homeostasis governed by cell type-specific and branched feedback loops acting on NMD. Mol Cell. 2011;43(6):950-61.

41. Chan WK, Huang L, Gudikote JP, et al. An alternative branch of the nonsense-mediated decay pathway. EMBO J. 2007;26(7):1820-30.

42. Schroeder A, Mueller O, Stocker S, et al. The RIN: an RNA integrity number for assigning integrity values to RNA measurements. BMC Mol Biol. 2006;7:3.

43. Bruning O, Rodenburg W, Radonic T, et al. RNA isolation for transcriptomics of human and mouse small skin biopsies. BMC Res Notes. 2011;4:438.

44. Yie SM, Lou B, Ye SR, et al. Detection of survivin-expressing circulating cancer cells (CCCs) in peripheral blood of patients with gastric and colorectal cancer reveals high risks of relapse. Ann Surg Oncol. 2008;15(11):3073-82.

45. Bertazza L, Mocellin S, Marchet A, et al. Survivin gene levels in the peripheral blood of patients with gastric cancer independently predict survival. J Transl Med. 2009;7:111.

46. Hoffmann AC, Warnecke-Eberz U, Luebke T, et al. Survivin mRNA in peripheral blood is frequently detected and significantly decreased following resection of gastrointestinal cancers. J Surg Oncol. 2007;95(1):51-4.

47. Terashima M, Yamakawa Y, Hatakeyama K, et al. Survivin expression in peripheral blood as a prognostic marker in patients with gastric cancer. J Clin Oncol 2014;32(suppl 15).

48. Black DL. Mechanisms of alternative pre-messenger RNA splicing. Annu Rev Biochem. 2003;72:291-336.

49. Birney E, Andrews D, Bevan P, et al. Ensembl 2004. Nucleic Acids Res 2004;32(database issue):D468-D470.

50. Birney E, Andrews TD, Bevan P, et al. An overview of Ensembl. Genome Res. 2004;14(5):925-8.

51. Furuta K, Arao T, Sakai K, et al. Integrated analysis of whole genome exon array and array-comparative genomic hybridization in gastric and colorectal cancer cells. Cancer Sci. 2012;103(2):221-7.

52. Hatakeyama K, Ohshima K, Fukuda Y, et al. Identification of a novel protein isoform derived from cancer-related splicing variants using combined analysis of transcriptome and proteome. Proteomics. 2011;11(11):2275-82.

53. Pastor F, Kolonias D, Giangrande $\mathrm{PH}$, et al. Induction of tumour immunity by targeted inhibition of nonsense-mediated mRNA decay. Nature (Lond). 2010;465(7295):227-30. 
54. Bonomi S, di Matteo A, Buratti E, et al. HnRNP A1 controls a splicing regulatory circuit promoting mesenchymal-to-epithelial transition. Nucleic Acids Res. 2013;41(18):8665-79.

55. Garcia-Olmo DC, Ruiz-Piqueras R, Garcia-Olmo D. Circulating nucleic acids in plasma and serum (CNAPS) and its relation to stem cells and cancer metastasis: state of the issue. Histol Histopathol. 2004;19(2):575-83.

56. Valadi H, Ekstrom K, Bossios A, et al. Exosome-mediated transfer of mRNAs and microRNAs is a novel mechanism of genetic exchange between cells. Nat Cell Biol. 2007;9(6):654-9.

57. Ohshima K, Inoue K, Fujiwara A, et al. Let-7 microRNA family is selectively secreted into the extracellular environment via exosomes in a metastatic gastric cancer cell line. PLoS One. 2010;5(10):e13247.

58. Meng S, Tripathy D, Shete S, et al. HER-2 gene amplification can be acquired as breast cancer progresses. Proc Natl Acad Sci USA. 2004;101(25):9393-8.

59. Gradilone A, Petracca A, Nicolazzo C, et al. Prognostic significance of survivin-expressing circulating tumour cells in T1G3 bladder cancer. BJU Int. 2010;106(5):710-5.

60. Krebs MG, Hou JM, Sloane R, et al. Analysis of circulating tumor cells in patients with non-small cell lung cancer using epithelial marker-dependent and -independent approaches. J Thorac Oncol. 2012;7(2):306-15.

61. Zhao L, Li P, Li F, et al. The prognostic value of circulating tumor cells lacking cytokeratins in metastatic breast cancer patients. J Cancer Res Ther. 2013;9(1):29-37.

62. Hosokawa M, Kenmotsu H, Koh Y, et al. Size-based isolation of circulating tumor cells in lung cancer patients using a microcavity array system. PLoS One. 2013;8(6):e67466.

63. Wang J, He H, Yu L, et al. HSF1 down-regulates XAF1 through transcriptional regulation. J Biol Chem. 2006;281(5):2451-9.

64. Zhang W, Guo Z, Jiang B, et al. Identification of a functional p53 responsive element within the promoter of XAF1 gene in gastrointestinal cancer cells. Int J Oncol. 2010;36(4):1031-7.

65. Leaman DW, Chawla-Sarkar M, Vyas K, et al. Identification of $\mathrm{X}$-linked inhibitor of apoptosis-associated factor-1 as an interferon-stimulated gene that augments TRAIL Apo2L-induced apoptosis. J Biol Chem. 2002;277(32):28504-11.
66. Chawla-Sarkar M, Lindner DJ, Liu YF, et al. Apoptosis and interferons: role of interferon-stimulated genes as mediators of apoptosis. Apoptosis. 2003;8(3):237-49.

67. Wang J, Peng Y, Sun YW, et al. All-trans retinoic acid induces $\mathrm{XAF} 1$ expression through an interferon regulatory factor-1 element in colon cancer. Gastroenterology. 2006;130(3):747-58.

68. Micali OC, Cheung HH, Plenchette S, et al. Silencing of the XAF1 gene by promoter hypermethylation in cancer cells and reactivation to TRAIL-sensitization by IFN-beta. BMC Cancer. 2007;7:52.

69. Reu FJ, Bae SI, Cherkassky L, et al. Overcoming resistance to interferon-induced apoptosis of renal carcinoma and melanoma cells by DNA demethylation. J Clin Oncol. 2006;24(23):3771-9.

70. Mitchell MJ, King MR. Computational and experimental models of cancer cell response to fluid shear stress. Front Oncol. 2013;3:44.

71. Gakhar G, Navarro VN, Jurish M, et al. Circulating tumor cells from prostate cancer patients interact with E-selectin under physiologic blood flow. PLoS One. 2013;8(12):e85143.

72. Kutun S, Celik A, Cem Kockar M, et al. Expression of CK-19 and CEA mRNA in peripheral blood of gastric cancer patients. Exp Oncol. 2010;32(4):263-8.

73. Schulze K, Gasch C, Staufer K, et al. Presence of EpCAMpositive circulating tumor cells as biomarker for systemic disease strongly correlates to survival in patients with hepatocellular carcinoma. Int J Cancer. 2013;133(9):2165-71.

74. Partridge M, Brakenhoff R, Phillips E, et al. Detection of rare disseminated tumor cells identifies head and neck cancer patients at risk of treatment failure. Clin Cancer Res. 2003;9(14):5287-94.

75. Hristozova T, Konschak R, Stromberger C, et al. The presence of circulating tumor cells (CTCs) correlates with lymph node metastasis in nonresectable squamous cell carcinoma of the head and neck region (SCCHN). Ann Oncol. 2011;22(8):1878-85.

76. Tsuji T, Ibaragi S, Hu GF. Epithelial-mesenchymal transition and cell cooperativity in metastasis. Cancer Res. 2009;69(18):7135-9. 